\title{
George Sand, la musica e i musicisti romantici, a cura di Mariantonietta Caroprese
}

\section{Annalisa Bottacin}

\section{(2) OpenEdition}

\section{Journals}

\section{Edizione digitale}

URL: http://journals.openedition.org/studifrancesi/5682

DOI: ERREUR PDO dans/localdata/www-bin/Core/Core/Db/Db.class.php L.34 : SQLSTATE[HY000]

[2006] MySQL server has gone away

ISSN: 2421-5856

\section{Editore}

Rosenberg \& Sellier

\section{Edizione cartacea}

Data di pubblicazione: 1 septembre 2011

Paginazione: 435-437

ISSN: 0039-2944

\section{Notizia bibliografica digitale}

Annalisa Bottacin, "George Sand, la musica e i musicisti romantici, a cura di Mariantonietta Caroprese», Studi Francesi [Online], 164 (LV | II) | 2011, online dal 30 novembre 2015, consultato il 07 janvier 2021. URL: http://journals.openedition.org/studifrancesi/5682 ; DOI: https://doi.org/10.4000/studifrancesi. 5682

Questo documento è stato generato automaticamente il 7 janvier 2021.

\section{cc) (†)}

Studi Francesi è distribuita con Licenza Creative Commons Attribuzione - Non commerciale - Non opere derivate 4.0 Internazionale. 


\title{
George Sand, la musica e i musicisti romantici, a cura di Mariantonietta Caroprese
}

\author{
Annalisa Bottacin
}

\section{NOTIZIA}

AA. VV., George Sand, la musica e i musicisti romantici, atti della giornata di studi (Latina, 21 sett. 2007), a cura di Mariantonietta CAROPRESE, Lucca, Biblioteca Musicale Italiana, 2010, VII-197 pp.

1 Nell'accostarsi al mondo di George Sand e alla sua poliedrica produzione letteraria, emerge un tema, quello relativo alla cultura musicale, che, come dimostrano gli atti di questo interessante convegno, doveva essere ancora una volta affrontato, contribuendo ad aumentare la conoscenza del rapporto musica/scrittura. Uno sguardo d'insieme sulla produzione sandiana fa ben rilevare quanto questi due universi si articolino tra loro. Per altro il fatto di avere avuto una relazione intima con chopin, che può ben definirsi un miracolo di musica assoluta, uomo discretissimo e compositore incomparabile, evidenzia in George Sand la capacità di comprendere generi diversi, dando prova di grande eclettismo in campo musicale.

2 Mariantonietta CAROPRESE, che firma oltre a un intervento anche la Prefazione al volume (pp XI-XIII), evidenza come sia stata profonda e costante la passione della scrittrice per la musica tanto da "poter vantare una formazione musicale che le consentì di scrivere dell'arte dei suoni con una certa competenza» (p. XI). L'incontro con Chopin non la trovò affatto impreparata, in quanto Aurore a Parigi già frequentava i milieux musicali e artistici.

Annarosa POLI concentra la sua attenzione in tema in un corposo articolo dal titolo: George Sand: una vita troppo nota, un'opera scoperta troppo tardi (pp. 4-18). L'eminente studiosa sandiana mette in luce, avvalendosi altresì di un excursus sull'opera della 
châtelaine de Nohant e su alcuni fondamentali passaggi della sua esistenza, quanto nell'educazione della scrittrice la musica abbia avuto un ruolo di spicco, insieme alla cultura classica, alle scienze naturali e all'equitazione. L'infelice matrimonio con il barone Casimir Dudevant, signorotto di campagna, e la susseguente rottura, la conducono a Parigi dove Sand ha un contatto più diretto con la musica e inizia la sua carriera di scrittrice con La Prima Donna. Conosce il melodramma italiano e «le divin Rossini», osannato anche da Stendhal che nel 1831 gli dedica la ben nota biografia, allorché l'artista era ancora vivente. Anche Rose et Blanche, il suo secondo romanzo, redatto anch'esso nel 1831, raccoglie molte impressioni nate dalle sue assidue frequentazioni di teatri parigini. L'amicizia con Liszt fu momento importante nella sua vita, in quanto il grande pianista non solo la introdusse nel mondo musicale parigino ma nel 1836 le presentò Chopin, che condurrà Sand a sempre più vicini precetti musicali. Come scrive Poli, la musica sarà ancora per Aurore conforto dopo la fine della liaison con Chopin; la ripresa dello studio del pianoforte l'aiuterà «a ritrovare il suo equilibrio interiore» (p. 16).

4 Anche Stefano RAGNI nella sua articolata comunicazione, "La Prima Donna" di George Sand: nasce il mito della 'diva' (pp. 19-45) si orienta su George Sand donna e artista, incentrando la sua attenzione su questo romanzo di argomento musicale, che inaugura il cammino scritturale di Sand. La Prima Donna apparve infatti sulla «Revue de Paris» nell'aprile del 1831; il pezzo, tanto apprezzato da Balzac, era firmato Jules Sand, e come rileva Annarosa Poli nel precedente articolo, era stato redatto a due mani con «un giovane studente di giurisprudenza, conosciuto in Berry: Jules Sandeau» (p. 5). In realtà sia le convenzioni letterarie dell'epoca, sia la proibizione della suocera, la baronessa Dudevant, di usare il nome di famiglia su qualsiasi scritto, costrinsero l'autrice all'uso di uno pseudonimo. Ciò che comunque emerge, è che «realmente sandiani nel vero senso del termine risultano il saliente narrativo e le componenti morfologiche della storia, vale a dire la musica operistica italiana e la voce umana» (pp. 19-20).

5 La relazione che ha come oggetto i rapporti di George Sand con i musicisti che più le furono vicini è firmata da Mariantonietta CAROPRESE, (George Sand e Franz Liszt tra Parigi, Gineva e Nohant (pp. 47-69). «Franz Liszt - esordisce Caroprese - fu il primo musicista che George Sand frequentò e il solo ad avere avuto con lei un profondo legame di amicizia, alimentato dagli stessi interessi e dalle stesse idee filosofiche, politiche e sociali» (p. 47). L'autrice percorre con sicurezza un cammino denso d'incontri e di situazioni, nello specifico, il rapporto con Franz Liszt, presentato a Aurore da Alfred de Musset nel 1834; Liszt, che ammirava gli scritti sandiani, la introdusse non solo nel milieu musicale ma altresì le fece conoscere le dottrine di Lammenais. Con Liszt la scrittrice instaurò un profondo rapporto di amicizia, che ebbe anche a creare una sorta di gelosia in Marie d'Agoult, all'epoca compagna del compositore, ma che fu fedele e duratura, come si può rilevare anche dalla loro correspondance.

6 La lunga amicizia, basata sulla reciproca stima, tra George Sand e il mezzosoprano Pauline Viardot-García, sorella della divina Malibran, è il soggetto del denso articolo di Raoul Meloncelli (La multiforme attività artistica di Pauline Viardot-García egeria di George Sand nel panorama musicale europeo dell'Ottocento, pp. 71-105), che ripercorrendo le tappe più salienti della vita della cantante, analizza gli ambienti musicali parigini di cui la stessa Sand faceva parte. Per altro Pauline nello studio del pianoforte ebbe come maestro Franz Liszt e fu vicina a Chopin. «La scrittrice-scrive Meloncelli-che considerava la musica la più sublime delle arti, e che fu subito affascinata dalla 
personalità della giovane artista in cui rivedeva l'immagine della sorella Maria [...] seguì poi con interesse e materna sollecitudine il progredire della sua carriera» (p. 73). $\mathrm{Fu}$ un rapporto che s'interromperà solo con la morte di Sand, che fu sempre vicina alla cantante, la cui ascesa, anche per l'inevitabile raffronto con il sempre vivo ricordo della sorella, non fu scevra da difficoltà e amarezze. In effetti Pauline è meno ricordata di Maria Malibran, scomparsa nel fiore degli anni, seppur sia morta ottantenne e sia stata l'acclamata interprete di un vastissimo repertorio. Essa fu anche attiva come compositrice, lasciò studi musicali, operine, trascrizioni e alcune melodie, di cui una su un testo di Musset.

7 L'articolata comunicazione di Danièle PISTONE, La musica notturna nella Francia romantica: da Parigi a Nohant (pp. 107-123), si orienta su un tema caro a George Sand, quello della notte, in cui la scrittrice era solita scrivere e creare, tanto «da costituire un tema fondamentale nella sua opera» (p. 107). In effetti ispirò Alfred de Musset, durante il loro soggiorno veneziano, per la creazione del ciclo delle celebri Nuits. Come nota l'autrice, il tema della notte nel romanticismo fu molto diffuso; nei racconti di E.T.A. Hoffmann, scrittore ben noto a Sand, è sovente accostato al tema della morte. Comunque in epoca romantica la musica notturna non è più quella delle serenate ma «trova piuttosto la sua origine nel notturno vocale, spesso cantato in duo» (p.109). Nella letteratura musicale del romanticismo il notturno fu una breve composizione prevalentemente pianistica di carattere melodico e sognante, introdotta da John Field, che raggiungerà il proprio acme con Fryderyk Chopin, autore di ventuno Notturni. Dall'anno 1836 fino al 1846 il compositore fu vicino a George Sand, che tra i primi seppe apprezzare le sue straordinarie composizioni a Parigi e a Nohant. L'intervento è corredato da sei Appendici (pp. 117-123).

8 Nell'ampia comunicazione, Fryderyk Chopin e George Sand: tra vita e arte, (pp. 125-68), Claudia Colombati presenta dettagliatamente quello che «fu un sodalizio più artistico che esistenziale, sia nelle visioni affini che nelle differenze» (p.125), allargando comunque il campo d'indagine sul versante della vita musicale parigina e su due luoghi "mitici": Majorca e Nohant. In effetti, rileva Colombati, se Parigi fu per il musicista luogo particolare e determinante per una prima svolta esistenziale, anche Majorca e poi Nohant funzionarono quale agenti provocatori della creazione artistica. Il contributo è suddiviso in vari sottocapitoletti: George Sand: "Essai sur le drame fantastique Goethe-ByronMickiewicz" (pp. 135-137), fa allusione a un saggio redatto da Sand a Majorca sul dramma metafisico, per altro di grande qualità, per la stesura del quale pare si sia avvalsa dell'aiuto di Chopin, in particolar modo nelle traduzioni dal polacco. In George Sand e Nohant (anni 1838-1847) (pp.137-144), viene analizzato il periodo assai fruttuoso di Chopin, benché molto malato, nella quiete del Berry e la comunanza di affetti e di intenti che era sempre più venuta a instaurarsi con la châtelaine de Nohant. Seguono ancora: A proposito di ambiguità (pp. 139-144); Parigi, George Sand, Franz Liszt e le eventuali influenze nella ricezione chopiniana dell'opera di L.van Beethoven (pp.144-150); L'arte: Fryderyk Chopin, Eugène Delacroix, George Sand (pp. 150-157) e "Lucrezia Floriani" e il periodo del distacco (pp. 157-168).

Markus ENGELHARDT in La lettera aperta di George Sand a Giacomo Meyerbeer (pp. 169-181) fa appunto riferimento alla lettera che il 15 novembre 1836 apparve sulla «Revue des deux mondes», indirizzata al principale esponente del «Grand opéra», e analizza le ragioni di tale documento, «prezioso anche per valutare la ricezione coeva di Mayerbeer e della forma individuale e innovativa che lui intendeva dare al teatro musicale nella capitale 
francese degli anni '30» (p. 169). Comunque l'incontro tra letteratura e musica non produsse i fini sperati né alcun risultato concreto, seppur tra Sand e Meyerbeer intercorse sincera e reciproca stima. Segue a chiusura del volume la bibliografia (pp. 183-190) e l'indice dei nomi (pp. 191-197). 